\title{
Error Exponents for Multi-tone Frequency Shift Keying on Wideband Rayleigh Fading Channels
}

\author{
Cheng Luo ${ }^{1}$, Muriel Médard ${ }^{1}$, Lizhong Zheng \\ Laboratory for Information and Decision Systems \\ Massachusetts Institute of Technology, Cambridge, MA, 02139 \\ Email: chengluo@mit.edu, medard@mit.edu, lizhong@mit.edu
}

\begin{abstract}
Flash signalling (with vanishing duty cycle) Frequency Shift Keying (FSK) is known to be a capacity-achieving modulation for multi-path fading channels in the limit of infinite bandwidth. However, since capacity-achieving schemes using flash FSK build the richness of their codebooks in frequency, the data rates of such schemes increase slowly with bandwidth. We seek to establish schemes that exhibit better performance than flash FSK for large but finite bandwidth. We consider Multi-tone FSK, which we have shown in our previous work that can also achieve the infinite-bandwidth capacity limit for multi-path fading channels, but is more general than FSK. Given its increased generality vis-à-vis FSK, and its optimality from a capacity point of view, Multi-tone FSK is a good candidate for transmission over very wide bandwidth. In this paper, we discuss upper and lower bounds of error probabilities for the family of Multi-tone FSK in Rayleigh fading channels. We find that these two bounds coincide in the infinite bandwidth limit and are therefore asymptotically tight. We compare the error probabilities of FSK and Multi-tone FSK in different situations and conclude that FSK is the preferable scheme when average power is the biting constraint, and Multi-tone FSK may be preferable when peak power is a limiting factor. We also explore the relationship among capacity and parameters related to time efficiency and spectrum efficiency.
\end{abstract}

\section{INTRODUCTION}

Spread-spectrum schemes which are adopted in the third generation of mobile communication systems are known to be not optimal in wideband applications, because their capacity decreases to zero as the bandwidth of system goes to infinity ( [1]). Results by Kennedy ( [2]), Gallager ( $[3, \S 8.6])$, Telatar, and Tse ( [4]) have shown that, with infinite bandwidth and fixed average received power, the capacity of a multipath fading channel is equal to that of an AWGN (additive white Gaussian noise) channel. The capacity-achieving scheme is a special FSK signalling which transmits in a flash (or impulsive) manner, i.e., symbols are sent in a small fraction of all symbol slots (low duty cycle) with a high peak power. In contrast to using signals that mimic white noise as in spreadspectrum scheme, this scheme is "peaky" both in time and frequency (a symbol occupies a small interval of bandwidth). Because these kinds of signalling have high peak power in a short time like flash, they are often called "flash signalling" in the literature.

\footnotetext{
${ }^{1}$ Supported by National Science Foundation, "CAREER", CCR-00993349 and Hewlett-Packard, "Advanced Concepts in Wireless Networking", 008542008
}

We have studied the capacity of FSK signalling in [5] for finite bandwidth and have shown that it can achieve performance of the order of infinite-bandwidth capacity in multi-path fading channels. However, even if the capacity grows linearly with power in the low SNR (signal-to-noise ratio) region, it will eventually be limited by bandwidth because it has low spectrum efficiency. Thus, we introduce a whole family of schemes which could give higher spectrum efficiency with the same capacity limit in infinite bandwidth. We call these schemes as Multi-tone FSK.

In Multi-tone FSK, we send several frequencies (tones) at a time while FSK sends only one tone. The signals are also transmitted in a small fraction of symbol slots. For transmitted symbols, each tone has equal transmission power. With the same number of total frequencies, the size of the symbol set for Multi-tone FSK is larger than that of FSK, which allows higher spectrum efficiency. We have shown that the infinite bandwidth capacity limit can be achieved by using Multi-tone FSK for an arbitrary number of tones. This result enriches the family of capacity achieving schemes. In particular, we have shown in [6] that two-tone FSK can outperform FSK in bandwidth-limited regions.

These results show that Multi-tone FSK is a promising modulation scheme and stimulate our further efforts. We seek to solve following questions: 1) what's the relationship between spectrum efficiency (related to the number of used tones) and system performance, 2) how do the parameters (symbol time, duty cycle, etc.) effect performance, and consequently 3) how should we select schemes for given power and bandwidth constraints?

To answer these questions, we study the error exponents of Multi-tone FSK in this paper. We derive an upper bound and a lower bound of error probability for Multi-tone FSK which are found to be coincident at the infinite bandwidth limit. So we can use these bounds to study the interplay amongst the error probability, bandwidth, data rate, and "peakiness" of the scheme. We use simulation to compare the performance of the schemes.

In Section II, the system model is built based on the Rayleigh fading channel assumption. In Section III, we give an upper bound of error probability for the Multi-tone FSK system. We derive a lower bound in Section IV. In Section $\mathrm{V}$, we illustrate the behavior of error probability in Multi-tone FSK systems comparing to that in FSK systems. 


\section{CAPACITY-ACHIEVING SCHEME}

In a multipath fading channel, the channel output $y(t)$ to an input waveform $x(t)$ is given by

$$
y(t)=\sum_{l=1}^{L} a_{l}(t) x\left(t-d_{l}(t)\right)+z(t),
$$

where $L$ is the number of paths, $a_{l}(t)$ and $d_{l}(t)$ are the gain and delay of the $l$ th path respectively at time $t$, and $z(t)$ is bandwidth-limited white Gaussian noise with power spectral density $N_{0} / 2$.

Let $T_{c}$ and $T_{d}$ be the coherence time and delay spread of the fading channel respectively. We assume that 1$)$ the processes $\left\{a_{l}(t)\right\}$ and $\left\{d_{l}(t)\right\}$ are i.i.d. and constant over time intervals of $T_{c}$ (block-fading model in time), and that 2) $T_{d} \ll T_{c}$ (an under-spread channel). Thus, the system model is

$$
y(t)=\sum_{l=1}^{L} a_{l} x\left(t-d_{l}\right)+z(t) .
$$

In FSK systems, we use sinusoids with different frequencies to represent symbols. Suppose that the average power constraint is $P$, system bandwidth is $B$, and the length of a symbol slot is $T_{s}$, which satisfies $T_{d}<T_{s} \leq T_{c}$. In order to keep all sinusoids orthogonal to each other on the symbol time $\left[T_{d}, T_{s}\right]$, the frequencies $f_{i}$ 's of sinusoids are chosen to be integer multiples of $1 /\left(T_{s}-T_{d}\right)$. Hence, we have $M=B /\left(T_{s}-T_{d}\right)$ frequencies in our system. Let us assume a $Q$-tone FSK scheme in which we use $Q$ tones to transmit a symbol. The system bandwidth is not changed with respect to FSK, but the size of input symbols is increased.

Let $\theta \in(0,1]$ be the duty cycle in the system, which means we transmit data for a fraction $\theta$ of time. By doing so, we raise the peak power of a sinusoid to $P / Q \theta$. One codeword is represented by $Q$ different complex sinusoids with the same amplitude $\sqrt{P / Q \theta}$. We define

$$
x(t)=\left\{\begin{array}{lll}
\sqrt{P / Q \theta} & \sum_{i \subseteq C_{m}} \exp \left(j 2 \pi f_{i} t\right) & 0 \leq t \leq T_{s}, \\
0 & \text { otherwise }
\end{array}\right.
$$

where $C_{m}$ is a set of $Q$ different integers in $\{1,2, \ldots, M\}(1 \leq$ $m \leq\left(\begin{array}{c}M \\ Q\end{array}\right)$ ). There are $\left(\begin{array}{c}M \\ Q\end{array}\right)$ combinations for the selection of $Q$ integers.

We consider the channel output over the interval $\left[T_{d}, T_{s}\right]$. During this interval, the $Q$ sinusoids have different channel gains and delays which are constant in the interval. We denote the gain and the delay for the $l$ th path of the $i$ th tones by $a_{l, i}$ and $d_{l, i}$ respectively. Hence by (2), when a message $m$ is sent, the received signal is

$$
\begin{aligned}
y(t) & =\sum_{i \subseteq C_{m}} \sum_{l=1}^{L} a_{l, i} \sqrt{P / Q \theta} \exp \left(j 2 \pi f_{i}\left(t-d_{l, i}\right)\right)+z(t) \\
& =\sum_{i \subseteq C_{m}} G_{i} \sqrt{P / Q \theta} \exp \left(j 2 \pi f_{i} t\right)+z(t)
\end{aligned}
$$

where $G_{i}=\sum_{l=1}^{L} a_{l, i} \exp \left(-j 2 \pi f_{i} d_{l, i}\right)$ is a complex-valued random variable. We define signal power in the conventional sense as the received signal power, and thus the channel gain can be normalized so that $E\left[\left|G_{i}\right|^{2}\right]=1$.

At the receiver, we correlate the received signal with all $M$ sinusoids, in which we can get the correlator outputs

$$
R_{k}=\frac{1}{\sqrt{N_{0} T_{s}^{\prime}}} \int_{T_{d}}^{T_{s}} \exp \left(-j 2 \pi f_{k} t\right) y(t) d t
$$

for $1 \leq k \leq M$, where $T_{s}^{\prime}=T_{s}-T_{d}$. Therefore,

$$
R_{k}= \begin{cases}G_{k} \sqrt{\frac{P T_{s}^{\prime}}{Q \theta N_{0}}}+W_{k} & k \subseteq C_{m}, \\ W_{k} & \text { Otherwise; }\end{cases}
$$

where $\left\{W_{k}\right\}$ is a set of i.i.d. circularly-symmetric complex Gaussian random variables, which satisfy $E\left[\left|W_{k}\right|^{2}\right]=1$.

The message is then repeated over $N$ disjoint time intervals to obtain time diversity. If no diversity is needed, we can let $N=1$. For $1 \leq k \leq M$ and $1 \leq n \leq N$, we have

$$
R_{k, n}= \begin{cases}G_{k, n} \sqrt{\frac{P T_{s}^{\prime}}{Q \theta N_{0}}}+W_{k, n} & k \subseteq C_{m}, \\ W_{k, n} & \text { Otherwise; }\end{cases}
$$

where $\left\{G_{k, n}\right\}$ is a set of i.i.d. complex random variables with $E\left[\left|G_{k, n}\right|^{2}\right]=1$ and $\left\{W_{k, n}\right\}$ is a set of i.i.d. circularlysymmetric complex Gaussian random variables of unit variance. We form the decision variables

$$
S_{k}=\frac{1}{N} \sum_{n=1}^{N}\left|R_{k, n}\right|^{2}
$$

and use the threshold decision rule with a threshold for simplicity. The threshold is

$$
A=1+(1-\epsilon) \frac{P T_{s}^{\prime}}{Q \theta N_{0}}
$$

where $\epsilon \in(0,1)$ may be freely chosen over its domain. If there are exact $Q$ of $S_{k}$ 's exceed the threshold, then we decode corresponding $m$; otherwise we declare an error. This decision scheme is asymptotically optimal.

The scheme transmits $\ln \left(\begin{array}{l}M \\ Q\end{array}\right)$ nats in $N T_{s} / \theta$ seconds, so the data rate $R$ is given by

$$
R=\frac{\theta}{N T_{s}} \ln \left(\begin{array}{c}
M \\
Q
\end{array}\right) .
$$

\section{UPPER BOUND ON THE ERROR PROBABILITY}

From the above detection scheme, an error occurs if $S_{m}<$ $A$ for $m \subseteq C_{m}$ or if $S_{l} \geq A$ for some $l \nsubseteq C_{m}$. Using the union bound, the symbol error probability can be bounded as

$$
\begin{aligned}
p_{e} & \leq \operatorname{Pr}\left\{\bigcup_{l \nsubseteq C_{m}} S_{l} \geq A\right\}+\operatorname{Pr}\left\{\bigcup_{m \subseteq C_{m}} S_{m}<A\right\} \\
& \leq(M-Q) \operatorname{Pr}\left\{S_{l} \geq A\right\}+Q \operatorname{Pr}\left\{S_{m}<A\right\} .
\end{aligned}
$$

We apply the Chernoff bound to obtain an upper bound for $\operatorname{Pr}\left\{S_{l} \geq A\right\}$ :

$$
\operatorname{Pr}\left\{S_{l} \geq A\right\} \leq \exp (-N \mathbf{E}(A))
$$


where

$$
\begin{aligned}
\mathbf{E}(A) & =\sup _{s}\left[s A-\ln \left(\mathbf{E}\left[\exp \left(s\left|W_{1,1}\right|^{2}\right)\right]\right)\right] \\
& =\sup _{s}[s A+\ln (1-s)] \\
& =A-1-\ln (A) .
\end{aligned}
$$

Hence, noting that $M-Q \leq Q\left(\begin{array}{c}M \\ Q\end{array}\right)^{1 / Q}$, we can bound the first term of right hand side in equation (11) as:

$$
\begin{aligned}
(M & -Q) \operatorname{Pr}\left\{S_{l} \geq A\right\} \leq Q\left(\begin{array}{c}
M \\
Q
\end{array}\right)^{1 / Q} \operatorname{Pr}\left\{S_{l} \geq A\right\} \\
& \leq Q \exp \left(-\frac{1}{Q} \ln \left(\begin{array}{c}
M \\
Q
\end{array}\right)\left[\frac{(1-\epsilon) P\left(1-T_{d} / T_{s}\right)}{R N_{0}}\right.\right. \\
& \left.\left.-1-\frac{Q \theta}{R T_{s}} \ln \left(1+\frac{(1-\epsilon) P T_{s}^{\prime}}{Q \theta N_{0}}\right)\right]\right) \\
& \triangleq p_{e, 1 u}(M, R, \theta, \epsilon) .
\end{aligned}
$$

All above results are based on general multipath fading assumption. To upper bound the second term of right hand side in (11), we assume that the fading is Rayleigh for simplicity. Under Rayleigh fading channel assumption, $G_{n}$ are i.i.d. circularly-symmetric complex Gaussian random variables. It follows that $\left|G_{n} \sqrt{P T_{s}^{\prime} /\left(Q \theta N_{0}\right)}+W_{k, n}\right|^{2}$ are i.i.d. exponentially distributed random variables with mean $P T_{s}^{\prime} /\left(Q \theta N_{0}\right)+1$. Applying the Chernoff bound and using (10) yields

$$
\begin{aligned}
& Q \operatorname{Pr}\left\{S_{m} \leq A\right\}=Q \operatorname{Pr}\left\{N S_{m} \leq N A\right\} \\
& \leq Q \exp \left(-N \sup _{s<0}\left\{s A-\ln \left(\mathbf{E}\left[\exp \left(s\left|Z_{i}+W_{i, n}\right|^{2}\right)\right]\right)\right\}\right) \\
& =Q \exp \left(-N \sup _{s<0}\left\{s A-\ln \left(1-\left[1+\frac{P T_{s}^{\prime}}{Q \theta N_{0}}\right] s\right)\right\}\right) \\
& =Q \exp \left(-\frac{\theta \ln \left(\begin{array}{c}
M \\
Q
\end{array}\right)}{R T_{s}}\left[\frac{-\epsilon P T_{s}^{\prime}}{Q \theta N_{0}+P T_{s}^{\prime}}\right.\right. \\
& \left.\left.\quad-\ln \left(1-\frac{\epsilon P T_{s}^{\prime}}{Q \theta N_{0}+P T_{s}^{\prime}}\right)\right]\right) \\
& \triangleq p_{e, 2 u}(M, R, \theta, \epsilon) .
\end{aligned}
$$

Using (11), (14), and (15), we can bound the symbol error probability

$$
p_{e} \leq \min _{\epsilon \in(0,1)}\left\{p_{e, 1 u}(M, R, \theta, \epsilon)+p_{e, 2 u}(M, R, \theta, \epsilon)\right\} .
$$

Now by choosing the parameter $\epsilon$, we can optimize performance. Noting that $p_{e, 1 u}$ is strictly increasing with $\epsilon$ while $p_{e, 2 u}$ is strictly decreasing, we choose $\epsilon$ so that $p_{e, 1 u}(M, R, \theta, \epsilon)=p_{e, 2 u}(M, R, \theta, \epsilon)$. It is satisfied when

$$
\begin{array}{r}
\epsilon=\epsilon_{0} \triangleq \frac{Q \theta N_{0}+P T_{s}^{\prime}}{P T_{s}^{\prime}}\left[1-\frac{R T_{s} N_{0}}{P T_{s}^{\prime}}\right. \\
\left.-\frac{Q \theta N_{0}}{P T_{s}^{\prime}} \ln \left(1+\frac{P T_{s}^{\prime}}{Q \theta N_{0}}\right)\right] .
\end{array}
$$

For $\epsilon$ in $(0,1)$, the data rate $R$ must be in the range

$$
0 \leq R<\frac{P T_{s}^{\prime}}{N_{0} T_{s}}-\frac{Q \theta}{T_{s}} \ln \left(1+\frac{P T_{s}^{\prime}}{Q \theta N_{0}}\right) .
$$

Substituting $\epsilon_{0}$ in (17) for $\epsilon$ in (16), we can upper bound (16) by

$$
\begin{aligned}
p_{e} & \leq 2 p_{e, 1 u}\left(M, R, \theta, \epsilon_{0}\right) \\
& =2 Q \exp \left(-\ln \left(\begin{array}{c}
M \\
Q
\end{array}\right) E_{r}(R, \theta)\right)
\end{aligned}
$$

where

$$
\begin{aligned}
& E_{r}(R, \theta)= \\
& \frac{\theta}{R T_{s}}\left\{\frac{R T_{s} N_{0}}{P T_{s}^{\prime}}+\frac{Q \theta N_{0}}{P T_{s}^{\prime}} \ln \left(1+\frac{P T_{s}^{\prime}}{Q \theta N_{0}}\right)-1\right. \\
& \left.-\ln \left(\frac{R T_{s} N_{0}}{P T_{s}^{\prime}}+\frac{Q \theta N_{0}}{P T_{s}^{\prime}} \ln \left(1+\frac{P T_{s}^{\prime}}{Q \theta N_{0}}\right)\right)\right\} .
\end{aligned}
$$

Expression (19) gives us a strict upper bound for symbol error probabilities in Multi-tone FSK. $M$ increases proportionally with bandwidth. As bandwidth grows to infinity, $M$ becomes infinite, the upper bound of error probability can be arbitrarily small if $E_{r}(R, \theta)>0$. From the definition of $E_{r}(R, \theta)$, we know that, as long as $R$ is in the range given by (18), $E_{r}(R, \theta)>0$ is satisfied.

Equation (18) gives us insights to understand the roles of $Q$ and $\theta$ in achieving capacity limit with infinite bandwidth. For data rates in the range defined by (18), we can use Multi-tone (or single-tone) FSK system to transmit data with arbitrarily small error probability when bandwidth is large enough. The first term $P T_{s}^{\prime} / N_{0} T_{s}$ in the upper limit is the capacity of AWGN channel considering the effect of the delay spread $T_{d}$. We can let the second term be very small so that the AWGN capacity limit can be achieved in Rayleigh fading channel. The result coincides with the capacity limit of Multi-tone FSK proved in [6].

For a given power constraint, to make the second term small, we can adjust $T_{s}, \theta$, and $Q$. Increasing $T_{s}$ will decrease the second term as long as the channel assumption $T_{s}<T_{c}$ is satisfied. As $T_{c}$ grows, $T_{s}$ can be increased such that $R$ approaches the infinite bandwidth capacity. This is consistent with the view that when $T_{c}$ approaches infinity, the fading channel is equivalent to an AWGN channel. Decreasing $\theta$ is another method to improve the system capacity. If we increase $Q$ (using Multi-tone FSK), $\theta$ should be decreased more than single-tone FSK in order to achieve the same system capacity limit. $\theta$ is a parameter of time efficiency, while $Q$ is related to frequency efficiency. They are interchangeable in the sense that $Q \theta$ together effect the second term. So when frequency efficiency is increased, we can decrease time efficiency to counteract the effect and vice versa.

\section{LOWER BOUND ON THE ERROR PROBABILITY}

In last section, we discussed an upper bound for error probability. In this section, we will derive a lower bound.

Substituting (7) into (8), we have

$$
S_{k}= \begin{cases}\frac{1}{N} \sum_{n=1}^{N}\left|G_{k, n} \sqrt{\frac{P T_{s}^{\prime}}{Q \theta N_{0}}}+W_{k, n}\right|^{2} & k \subseteq C_{m}, \\ \frac{1}{N} \sum_{n=1}^{N}\left|W_{k, n}\right|^{2} & \text { Otherwise. }\end{cases}
$$


$S_{k}$ is a $\chi^{2}$ random variable with $2 N$ degrees of freedom, because $G_{k, n}$ and $W_{k, n}$ are i.i.d circularly symmetric complex Gaussian random variables of unit variance. The cumulative distribution function of a $\chi^{2}$ random variable with $2 N$ degrees of freedom can be exactly evaluated ( $[9, \S 2.1 .4])$.

Define

$$
\begin{aligned}
p_{e, 1 l} & \triangleq \operatorname{Pr}\left\{\sum_{n=1}^{N}\left|G_{k, n} \sqrt{\frac{P T_{s}^{\prime}}{Q \theta N_{0}}}+W_{k, n}\right|^{2}<N A\right\} \\
& =\exp \left\{-\frac{N A}{1+\frac{P T_{s}^{\prime}}{Q \theta N_{0}}}\right\} \sum_{k=N}^{\infty} \frac{1}{k !}\left(\frac{N A}{1+\frac{P T_{s}^{\prime}}{Q \theta N_{0}}}\right)^{k} \\
& =\exp \left\{-N A^{\prime}\right\} \sum_{k=N}^{\infty} \frac{\left(N A^{\prime}\right)^{k}}{k !}
\end{aligned}
$$

where $A^{\prime}=\frac{A}{1+P T_{s}^{\prime} /\left(Q \theta N_{0}\right)}$. Because $\left(N A^{\prime}\right)^{k} / k !$ is positive for all $\mathrm{k}$, we have

$$
\sum_{k=N}^{\infty} \frac{\left(N A^{\prime}\right)^{k}}{k !} \geq \frac{\left(N A^{\prime}\right)^{N}}{N !},
$$

Therefore, we can lower bound $p_{e, 1 l}$ by applying (23) in (22)

$$
p_{e, 1 l} \geq \exp \left\{-N A^{\prime}+\ln \left[\frac{\left(N A^{\prime}\right)^{N}}{N !}\right]\right\} .
$$

Using Stirling's approximation $\sqrt{2 \pi N} N^{N} e^{\left(-N+\frac{1}{12 N+1}\right)}<$ $N !<\sqrt{2 \pi N} N^{N} e^{\left(-N+\frac{1}{12 N}\right)}$, we then obtain

$$
p_{e, 1 l}>\exp \left\{-N\left(A^{\prime}-1-\ln \left(A^{\prime}\right)+o_{1}(N)\right)\right\}
$$

where

$$
o_{1}(N)=\frac{1}{2 N} \ln (2 \pi N)+\frac{1}{12 N^{2}}
$$

goes to zero with increasing $N$.

Similarly, we define

$$
\begin{aligned}
p_{e, 2 l} & \triangleq \operatorname{Pr}\left\{\sum_{n=1}^{N}\left|W_{k, n}\right|^{2}>N A\right\} \\
& =\exp \{-N A\} \sum_{k=0}^{N-1} \frac{(N A)^{k}}{k !} .
\end{aligned}
$$

Because $(N A)^{k} / k$ ! is positive for all $\mathrm{k}$, we have

$$
\sum_{k=0}^{N-1} \frac{(N A)^{k}}{k !} \geq \frac{(N A)^{N-1}}{(N-1) !} .
$$

Therefore,

$$
p_{e, 2 l} \geq \exp \left\{-N A+\ln \left[\frac{(N A)^{N-1}}{(N-1) !}\right]\right\} .
$$

Applying Stirling's approximation, we then obtain

$$
p_{e, 2 l}>\exp \left\{-N\left(A-1-\ln (A)+o_{2}(N)\right)\right\}
$$

where $o_{2}(N)$ is given by

$$
o_{2}(N)=\frac{1}{2 N} \ln \left(2 \pi N A^{2}\right)+\frac{1}{12 N^{2}}
$$

which goes to zero with increasing $N$.

We now observe that

$$
\begin{aligned}
p_{e} & \geq \sum \operatorname{Pr}(\text { one error })-\sum \operatorname{Pr}(\text { two errors }) \\
& \geq Q p_{e, 1 l}+(M-Q) p_{e, 2 l}-(M-Q) Q p_{e, 1 u} p_{e, 2 u} \\
& -\frac{(M-Q)(M-Q-1)}{2} p_{e, 2 u}{ }^{2}-\frac{Q(Q-1)}{2} p_{e, 1 u}{ }^{2}
\end{aligned}
$$

which is a lower bound for error probability. In the discussion of the upper bound, we know that $M p_{e, 1 u}$ and $M p_{e, 2 u}$ are of the same order, and decrease to zero with increasing $M$. Hence, when we discuss the lower bound (32) in the infinite bandwidth condition, we can simply omit the last three terms. If $p_{e, 1 l} \geq p_{e, 2 l}$, then we have $Q p_{e, 1 l}+(M-Q) p_{e, 2 l} \geq M p_{e, 2 l}$; otherwise, $Q p_{e, 1 l}+(M-Q) p_{e, 2 l} \geq Q p_{e, 1 l}$. Consequently, we get two lower bounds for $p_{e}$ when $N \rightarrow \infty$, one is $Q p_{e, 1 l}$, the other is $M p_{e, 2 l}$. We obtain

$$
\begin{aligned}
& \lim _{N \rightarrow \infty} \frac{-\ln p_{e}}{N} \\
\leq & \min \left(\lim _{N \rightarrow \infty} \frac{-\ln \left(Q p_{e, 1 l}\right)}{N}, \lim _{N \rightarrow \infty} \frac{-\ln \left(M p_{e, 2 l}\right)}{N}\right) \\
\leq & \min \left(\lim _{N \rightarrow \infty} \frac{-\ln \left(p_{e, 1 l}\right)}{N}, \lim _{N \rightarrow \infty} \frac{-\ln \left(M p_{e, 2 l}\right)}{N}\right) \\
& \text { using the inequality } M<\left(\begin{array}{c}
M \\
Q
\end{array}\right)^{1 / Q} \\
\leq & \min \left(\lim _{N \rightarrow \infty} \frac{-\ln \left(p_{e, 1 l}\right)}{N}, \lim _{N \rightarrow \infty} \frac{-\ln \left(\left(\begin{array}{c}
M \\
Q
\end{array}\right)^{1 / Q} p_{e, 2 l}\right)}{N}\right) \\
& \text { using }(25) \text { and }(30) \\
\leq & \min \left(A^{\prime}-1-\ln \left(A^{\prime}\right), A-1-\ln (A)-R T_{s} /(Q \theta)\right) \\
= & \frac{R T_{s}}{\theta} E_{r}(R, \theta) .
\end{aligned}
$$

For $\epsilon$ in its allowable range (0,1), $R$ should satisfy (18). We further have

$$
\begin{aligned}
\lim _{N \rightarrow \infty} \frac{-\ln \left[p_{e} /(2 Q)\right]}{\ln \left(\begin{array}{c}
M \\
Q
\end{array}\right)} & =\lim _{N \rightarrow \infty} \frac{-\theta \ln \left[p_{e} /(2 Q)\right]}{N R T_{s}} \\
& =\lim _{N \rightarrow \infty} \frac{-\theta \ln \left(p_{e}\right)}{N R T_{s}} \leq E_{r}(R, \theta) .
\end{aligned}
$$

This upper bound for $\lim _{N \rightarrow \infty}\left(-\ln \left[p_{e} /(2 Q)\right]\right) / \ln \left(\begin{array}{c}M \\ Q\end{array}\right)$ is equivalent to the lower bound of error probability when $N$ goes to infinity. The reverse inequality follows from (19), the upper bound of error probability. Hence, we have

$$
\lim _{N \rightarrow \infty} \frac{-\ln \left[p_{e} /(2 Q)\right]}{\ln \left(\begin{array}{c}
M \\
Q
\end{array}\right)}=E_{r}(R, \theta) .
$$

Therefore $E_{r}(R, \theta)$ represents the true exponential dependence of the error probability on $\ln \left(\begin{array}{c}M \\ Q\end{array}\right)$ for $M$ sufficiently large.

Letting $Q=1$ in (19), and (35), we can get the results presented in [8] and [7]. So these results derived in this paper are more general versions, which are valid both for FSK and Multi-tone FSK systems. 


\section{NUMERICAL RESULTS}

To illustrate in a straightforward form, we plot simulation results in Figure 1 . We let $T_{s}=1 \mu s, T_{d}=0.1 \mu s, P=1000$, $N_{0}=1, R=800$ nats $/ \mathrm{sec}$ and study different upper bounds and lower bounds for $Q=1$ (single-tone FSK), $Q=2$ (two-tone FSK), and $Q=4$ (four-tone FSK) when we increase the total number of available tones $M$, which equals to increase the system bandwidth $B$ because $B=M / T_{s}^{\prime}$. We can find in (18) that $Q \theta$ determines how close the data rate can get to the upper limit $P T_{s}^{\prime} / N_{0} T_{s}$. Furthermore, to keep $Q \theta$ unchanged, we have the same peak power in these schemes when the average power is the same. Thus, we choose $\theta=0.01,0.005,0.0025$ for $Q=1,2,4$ respectively so that $Q \theta$ is a constant.

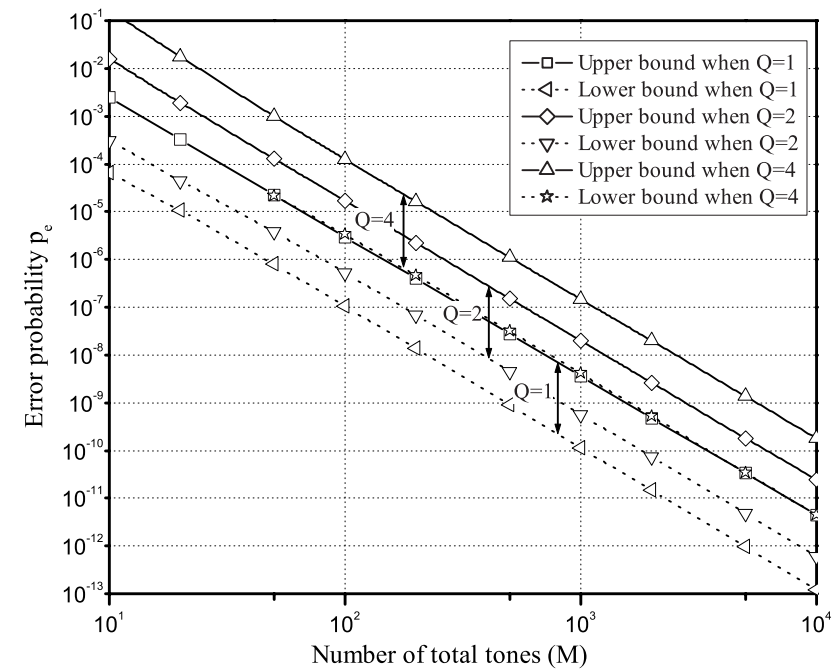

Fig. 1. Upper and lower bounds of error probability for single-tone FSK, two-tone FSK, and four-tone FSK when $Q \theta$ is fixed and system bandwidth is increasing.

In Figure 1, error probabilities decrease with $M$ when we use data rates smaller than the upper limit in (18). The difference between upper bounds and lower bounds goes to zero, which shows these two bounds are asymptotically tight. Under the same power and data rate condition, the error probabilities of Multi-tone FSK are higher than single-tone FSK. The more tones are used in transmission, the higher is error probability. However, it does not mean that Multi-tone FSK is worse than FSK.

In systems with peak power constraint, Multi-tone FSK will have advantages over FSK. In figure 2, we compare the performance for FSK, two-tone FSK, and three-tone FSK under this assumption. By letting $P=1000,2000,3000$ for $Q=1,2,3$ respectively, we keep the peak power and the duty cycle constant. The data rates are $R=20$ nats $/ \mathrm{sec}$.

The results show that the upper bounds of error probability for Multi-tone FSK are lower than that for FSK. We know these upper bounds are tight in the large bandwidth regime. Hence Multi-tone FSK outperforms FSK in this peak power constrained situation. Actually, Multi-tone FSK will also perform better than FSK in the bandwidth-limited channel. The performance comparison can be found in [10].

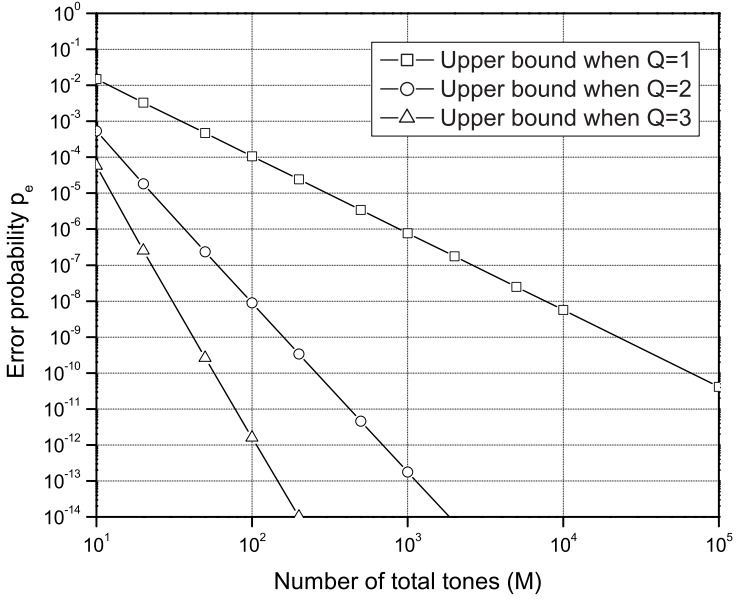

Fig. 2. Upper bounds of error probability for single-tone FSK, two-tone FSK, and three-tone FSK when peak power and duty cycle are fixed, and bandwidth is increasing.

\section{CONCLUSION}

In this paper, we obtain upper and lower bounds for Multitone FSK and derive the error exponent which represents the true exponential dependence of the error probability on the system bandwidth. We discuss the relationship among capacity, frequency and time efficiency and compare the performance of FSK and Multi-tone FSK. We conclude that FSK is the preferable scheme when average power is the active constraint, and Multi-tone FSK may be preferable when peak power is a limiting factor.

\section{ACKNOWLEDGMENT}

The authors would like to thank Desmond Lun for valuable discussions.

\section{REFERENCES}

[1] M. Médard, R. G. Gallager Bandwidth Scaling for Fading Multipath Channels. IEEE Transactions on Information Theory, 48(4):840-852, April, 2002.

[2] R. S. Kennedy. Fading Dispersive Communication Channels. Wiley Interscience, New York, NY, 1969.

[3] R. G. Gallager. Information Theory and Reliable Communication. John Wiley \& Sons, New York, NY, 1968.

[4] I. E. Telatar and D. N. C. Tse. Capacity and mutual information of wideband multipath fading channels. IEEE Trans. Inform. Theory, 46(4):1384-1400, July 2000.

[5] C. Luo, M. Médard. Frequency-shift keying for Ultrawideband - How close to capacity can we get? In Proc. of ISITA, October 2002.

[6] C. Luo, M. Médard. Frequency-shift Keying for Ultrawideband Achieving Rates of the Order of Capacity. In The 40th Annual Allerton Conference on Communication, Control, and Computing, October 2002.

[7] D. S. Lun, M. Médard, and I. C. Abou-Faycal. Error exponents for capacity-achieving signaling on wideband rayleigh fading channels. In International Symposium on Information Theory and its Applications, October 2002.

[8] D. S. Lun, M. Médard, and I. C. Abou-Faycal. Error exponents for wideband multipath fading channels - a strong coding theorem. In Proc. of Conference on Information Sciences and Systems (CISS), March 2002.

[9] J. G. Proakis. Digital Communications. McGraw-Hill, New York, NY, third edition, 1995.

[10] C. Luo, M. Médard. Performance of Single-tone and Two-tone Frequency-shift Keying for Ultrawideband. In 36th Asilomar Conference on Signals, Systems and Computers, Nov. 2002.

[11] S. Verdú. Spectral efficiency in the wideband regime. IEEE Trans. Inform. Theory, 48(6):1319 -1343, June 2002. 